ARTÍCULO

\title{
Efecto de la temperatura sobre la tasa de consumo del vitelo y gota de aceite en las larvas del pargo flamenco Lutjanus guttatus (Perciformes: Lutjanidae)
}

Effect of temperature on yolk and oil droplet consumption by spotted rose snapper larvae Lutjanus guttatus (Perciformes: Lutjanidae)

\section{María Isabel Abdo-de la Parra ${ }^{1,2, *}$, Gustavo Alejandro Rodríguez-Montes de Oca ${ }^{3}$, L. Estela Rodríguez-Ibarra ${ }^{1}$, José Cristóbal Román-Reyes ${ }^{3}$ y Gabriela Velasco-Blanco ${ }^{1}$}

\author{
${ }^{1}$ Centro de Investigación en Alimentación y Desarrollo, A.C., Av. Sábalo-Cerritos S/N, CP 82010, Mazatlán, Sinaloa, México \\ ${ }^{2}$ Posgrado de Ciencias Agropecuarias, Colegio de Ciencias Agropecuarias de la Universidad Autónoma de Sinaloa, Km. 17,5 Carretera \\ Culiacán-Dorado, CP 8000 Culiacán, Sinaloa, México \\ ${ }^{3}$ Laboratorio de Reproducción y Cultivo de Peces, Facultad de Ciencias del Mar, Universidad Autónoma de Sinaloa, Paseo Claussen \\ s/n, C.P. 82000 , Mazatlán, Sinaloa, México \\ *Autor corresponsal: abdo@ciad.mx
}

\begin{abstract}
The yolk is the main energy source for eggs and marine fish larvae. Temperature is a key factor in determining the efficiency of yolk conversion into body tissues, which can affect the size larvae at first feeding. In this work, the effect of different temperatures on the yolk sac consumption and oil droplet by the spotted rose snapper, Lutjanus guttatus was evaluated, in order to determine the adequate culture temperature during the first days after hatch. The fertilized eggs were placed in $20 \mathrm{~L}$ plastic tanks at different temperatures $\left(22,25,28\right.$ and $\left.31^{\circ} \mathrm{C}\right)$ with three replicates per treatment. The time and percentage of hatching, larvae total length, yolk consumption (TCV) and oil droplet (TCGA) rates at $0,24,48$ and $72 \mathrm{~h}$ after hatching (HDE) were determined. Eggs incubated at $22{ }^{\circ} \mathrm{C}$ did not hatch, while hatching time in eggs incubated at $31^{\circ} \mathrm{C}$ decreased significantly. No differences were observed in hatching percentage between treatments of temperature. Larvae reared at $31^{\circ} \mathrm{C}$ obtained the higher length value than those reared at $25^{\circ} \mathrm{C}$ at 0 and $24 \mathrm{HDE}$. The yolk and oil droplet were consumed faster by larvae reared at $31^{\circ} \mathrm{C}$ at 0 and $24 \mathrm{HDE}$. Total mortality was registered for larvae reared at 25 and $31{ }^{\circ} \mathrm{C}$ at 48 and $72 \mathrm{HDE}$, respectively. The optimal temperature for egg incubation and newly-hatched $\mathrm{L}$. guttatus larvae was $28^{\circ} \mathrm{C}$, which would favorably impel the consumption of energy reserves for its growth and survival.
\end{abstract}

Key words: Snapper larvae, hatch, growth, energy reserves

\begin{abstract}
Resumen.- El vitelo es la principal fuente de energía para huevos y larvas vitelinas de peces marinos. La temperatura tiene un papel dominante en la eficiencia de la conversión del vitelo en tejido corporal y afecta el tamaño que puede alcanzar la larva en la primera alimentación. En el presente trabajo se evaluó el efecto de diferentes temperaturas sobre el consumo del saco vitelino y la gota de aceite de pargo flamenco, Lutjanus guttatus, para determinar la temperatura adecuada de cultivo para larvas vitelinas. Los huevos fertilizados de pargo flamenco se colocaron en tanques de plástico de $20 \mathrm{~L}$ a diferentes temperaturas $\left(22,25,28\right.$ y $\left.31{ }^{\circ} \mathrm{C}\right)$ con tres réplicas por tratamiento. Se determinó el tiempo y porcentaje de eclosión, longitud total, tasas de consumo de vitelo (TCV) y de gota de aceite (TCGA) a las 0, 24, 48 y 72 h después de la eclosión (HDE). Los huevos incubados a $22^{\circ} \mathrm{C}$ no eclosionaron. No se observaron diferencias significativas en el porcentaje de eclosión entre tratamientos de 25,28 y $31^{\circ} \mathrm{C}$. Las larvas a $31^{\circ} \mathrm{C}$ presentaron mayor longitud al eclosionar y menor HDE que las que estuvieron a $25^{\circ} \mathrm{C}$. La TCV y TCGA fue mayor en las larvas a $31^{\circ} \mathrm{C}$ a 0 y $24 \mathrm{HDE}$. Se presentó mortalidad total en las larvas incubadas a 25 y $31^{\circ} \mathrm{C}$ a las 48 y $72 \mathrm{HDE}$, respectivamente. La temperatura adecuada para la incubación de huevos y larvas de $L$. guttatus se debe llevar a cabo a $28^{\circ} \mathrm{C}$ para favorecer el consumo de sus reservas energéticas adecuado para su crecimiento y supervivencia.
\end{abstract}

Palabras clave: Larvas de pargo flamenco, eclosión, crecimiento, reservas energéticas

\section{INTRODUCCIÓN}

En los huevos de los peces teleósteos, como el pargo flamenco Lutjanus guttatus (Steindachner, 1869), se forma un saco vitelino extraembrionario y se consideran como un sistema semicerrado donde solo se lleva a cabo el intercambio de gases (Heming \& Buddington 1988); por lo tanto, el vitelo es la principal fuente de energía, de hormonas y enzimas durante la embriogénesis y el periodo de alimentación endógena de las larvas (Kamler 1992, 2008). Los principales componentes del vitelo son las plaquetas de vitelo ricas en lipoproteínas y fosfoproteínas y las gotas de aceite que son principalmente lípidos neutrales con una alta proporción de ácidos grasos mono insaturados (Heming \& Buddington 1988). La tasa de absorción del vitelo y eficiencia de utilización de las reservas vitelinas, son indicadores que permiten determinar el desarrollo temprano, crecimiento y supervivencia de las larvas hasta el inicio de la alimentación exógena (Heming \& Buddington 1988). Al periodo de tiempo que comprende el paso de la alimentación endógena a exógena de las larvas se le conoce 
como "alimentación mixta" o "periodo crítico", tiempo en el cual la mortalidad puede ser muy alta (Hardy \& Livak 2004). La tasa de absorción del vitelo es baja al principio de la ontogenia y se incrementa poco a poco. Después de la eclosión, la absorción es más rápida que en los embriones (Kamler 1992), debido a que las larvas presentan tasas metabólicas más altas. La tasa absoluta de utilización de vitelo disminuye después del estadio de nado como resultado de una cantidad limitada de contenido de vitelo (Kamler 1992, Jaroszewska \& Dabrowski 2011).

Se ha demostrado que tanto la eficiencia, como la tasa de consumo del vitelo y de las gotas de aceite son específicas de cada especie y dependen también, de factores abióticos como la temperatura, luz, concentración de oxígeno disuelto en el agua y la salinidad (Blaxter 1992, Jaroszewska \& Dabrowski 2011); siendo la temperatura el factor más importante (Kamler 2002, Pittman et al. 2013). La temperatura también tiene un rol dominante en la eficiencia de la conversión del vitelo en tejido corporal y afecta el tamaño que puede alcanzar la larva en la primera alimentación (Kamler 2008) debido a su importancia en el control de los procesos fisiológicos (Blaxter 1992). El consumo lento o rápido del vitelo puede ser determinante en el desarrollo y supervivencia de las larvas. Se ha demostrado que la eficiencia en el consumo de las reservas energéticas larvarias es mayor en peces marinos cultivados dentro de los rangos óptimos de temperatura de cada especie, y disminuye cuando se cultivan fuera del rango óptimo (Kaminski et al. 2006, Peña et al. 2014, Barón-Aguilar et al. 2015, EstradaGodínez et al. 2015, Hui et al. 2015, Guevara-Fletcher et al. 2017). A temperaturas mayores del gradiente óptimo las larvas eclosionan en estadios prematuros y presentan sacos vitelinos más pequeños (Yoseda et al. 2006, Azocar et al. 2014); mientras que a gradientes inferiores se incrementa la incidencia de deformidades y altas mortalidades antes de completar la reabsorción del saco vitelino (Das et al. 2006, Brown et al. 2011, Pittman et al. 2013). Por lo anterior es necesario determinar experimentalmente la temperatura adecuada para favorecer de manera eficiente la reabsorción del vitelo en las larvas de peces marinos.

Hasta la fecha, una de las dificultades para el cultivo del pargo flamenco, a gran escala en México, es la producción masiva de juveniles de calidad (Álvarez-Lajonchère et al. 2012). En el Centro de Investigación en Alimentación y Desarrollo (CIAD), Mazatlán, México, se ha estudiado la larvicultura del pargo flamenco durante la última década; sin embargo, se ha registrado con frecuencia, alta mortalidad larvaria en la transición de la alimentación endógena a la exógena.

En el presente trabajo se evaluó el efecto de diferentes temperaturas sobre el consumo y tasa de absorción del saco vitelino y la gota de aceite de L. guttatus, para determinar la temperatura adecuada de cultivo para las larvas vitelinas de esta especie y aumentar la supervivencia de las mismas durante el periodo de alimentación endógena.

\section{MATERIALES Y MÉTODOS}

\section{OBTENCIÓN DE LOS HUEVOS FERTILIZADOS}

Los huevos fertilizados de pargo flamenco se obtuvieron de la planta piloto para la producción de peces marinos del CIAD, Unidad Mazatlán, mediante los protocolos establecidos por Ibarra-Castro \& Álvarez-Lajonchère (2011).

\section{DISEÑO EXPERIMENTAL}

Se evaluaron 4 temperaturas constantes $(22,25,28$ y $31{ }^{\circ} \mathrm{C}$ ) con 3 réplicas cada una. Se colocaron $2 \mathrm{~mL}$ de huevos fertilizados de pargo flamenco (alrededor de 2.500 huevos) en tanques de plástico transparente conteniendo $15 \mathrm{~L}$ de agua de mar filtrada por UV y se incubaron a la temperatura establecida. Los tanques del tratamiento de $22{ }^{\circ} \mathrm{C}$ se mantuvieron en ambiente climatizado a dicha temperatura, mientras que el resto de los tratamientos fueron colocados en un laboratorio climatizado a $25{ }^{\circ} \mathrm{C}$, mientras que para obtener los gradientes experimentales de 28 y $31{ }^{\circ} \mathrm{C}$ se utilizó un termostato (Termal, 300 watts) en cada contenedor, el cual se reguló para obtener la temperatura establecida. Cada réplica fue provista de aireación para mantener el oxígeno disuelto en el agua a 5 ppm. La temperatura y el oxígeno disuelto en el agua fueron monitoreados cada $4 \mathrm{~h}$ en todos los tanques con el equipo YSI modelo 55/12FT. El experimento se desarrolló sin recambio de agua; solo en el caso de evaporación para las más altas temperaturas experimentales, se agregó agua de mar filtrada.

\section{PorCentaJe Y TIEMPO DE ECLOSIÓN}

Se determinó el tiempo de eclosión por observación directa cada 20 min de cada una de las réplicas y el porcentaje de eclosión $(\% \mathrm{E})$ mediante la fórmula:

$\% \mathrm{E}=$ Larvas eclosionadas $/$ huevos incubados $* 100$

\section{CRECIMIENTO, TASA DE CONSUMO DEL VITELO Y DE LA GOTA DE ACEITE}

Se tomaron 20 larvas en 4 tiempos post-eclosión: al eclosionar $(0$ h) y a 24,48 y 72 h después de la eclosión (HDE) por cada réplica y en cada uno los tratamientos realizados. Para determinar la longitud total (LT), el volumen del saco vitelino $\left(\mathrm{VSV} \mathrm{mm}^{3}=(\pi / 6) \mathrm{LH}^{2}\right.$, donde $\mathrm{L}$ es la longitud y $\mathrm{H}$ es el ancho del saco vitelino, Kamler 2008) y el volumen de la gota de aceite $\left(V_{G A ~ m m}^{3}=4 / 3 \pi r^{3}\right.$, donde $\mathrm{r}$ es el radio de la gota lipídica, Williams et al. 2004), 
cada larva muestreada se anestesió con 2-fenoxietanol a una concentración de $0,1 \mathrm{ml} \mathrm{L}^{-1}$ y se colocó, mediante una pipeta Pasteur, en una laminilla excavada para obtener mediante un microscopio Olympus ${ }^{\circledR} \mathrm{CX} 31$ (4X con un micrómetro ocular de $25 \mu \mathrm{m}$ ) las medidas mencionadas. Para determinar la tasa de consumo del vitelo y de la gota de aceite se aplicó la siguiente fórmula: $\mathrm{TCV}$ y $\mathrm{TCGA}=(\mathrm{Vi}-\mathrm{Vf}) / \mathrm{Vi} * 100$, donde TCV o TCGA es la tasa de consumo final, Vi es el volumen inicial al eclosionar y $\mathrm{Vf}$ es el volumen final a un tiempo determinado (Hui et al. 2015).

Los resultados expresados porcentualmente fueron transformados a arcoseno para su posterior análisis. A todos los resultados se les determinó la normalidad (prueba de Bartlett) y la homocedasticidad (prueba de Levene); los datos normales se analizaron mediante el análisis de varianza de una vía (ANDEVA, $P<0,05$ ) y las diferencias significativas entre los tratamientos se determinaron por la prueba de comparación múltiple de rangos de Tukey $(P<0,05)$ (Zar 1996). Se identificaron los modelos de regresión que se ajustaron mejor a la relación empírica entre el consumo del vitelo y de la gota de aceite para cada temperatura, a través del tiempo (HDE). Los análisis estadísticos se llevaron a cabo mediante el programa STATGRAPHICS Plus 5.1 (Statpoint Technologies, Inc.)

\section{Resultados}

\section{TIEMPO Y PORCENTAJE DE ECLOSIÓN}

En los huevos incubados a $22{ }^{\circ} \mathrm{C}$ no hubo eclosión, los embriones se desarrollaron hasta la etapa de formación de órganos y cola, pero no eclosionaron. En el resto de los tratamientos evaluados, el porcentaje de eclosión no fue estadísticamente diferente $\left(\mathrm{F}_{(2,8)}=1,21, P=0,361\right)$ (Tabla
1). El tiempo de eclosión presentó diferencias significativas $\left(\mathrm{F}_{(2,8)}=92,33, P<0,001\right)$ el cual disminuyó en función del aumento de temperatura, las larvas incubadas a $31{ }^{\circ} \mathrm{C}$ eclosionaron a las $13 \mathrm{~h}$ después de la fertilización (HDF), mientras que las larvas incubadas a $25^{\circ} \mathrm{C}$ eclosionaron a las 19 HDF (Tabla 1).

\section{CRECIMIENTO Y CONSUMO DEL SACO VITELINO Y DE LA GOTA DE ACEITE}

Las larvas recién eclosionadas $(0 \mathrm{HDE})$ a $31^{\circ} \mathrm{C}$ presentaron la LT mas alta $\left(\mathrm{F}_{(2,97)}=4,40, P<0,05\right)$, tendencia que se observó también después de las 24 y 48 HDE (Tabla 1), las larvas recién eclosionadas a $28^{\circ} \mathrm{C}$ no presentaron diferencias significativas en su LT con las larvas del tratamiento de $25^{\circ} \mathrm{C}(P<0,05)$. Se registró el $100 \%$ de mortalidad de las larvas incubadas a 25 y $31{ }^{\circ} \mathrm{C}$ después de las 48 y $72 \mathrm{HDE}$, respectivamente (Tabla 1 ).

La absorción de las reservas endógenas fue mayor con el incremento de la temperatura (Tabla 2); el VSV y VGA en las larvas mantenidas a $31^{\circ} \mathrm{C}$, tanto al eclosionar como a las $24 \mathrm{HDE}$ fue significativamente menor $(P<0,05)$ a las larvas eclosionadas y mantenidas a 28 y $25^{\circ} \mathrm{C}$; mientras que a las $48 \mathrm{HDE}$ no se presentaron diferencias significativas $(P$ $>0,05)$ en el VSV y VGA entre las larvas cultivadas a 28 y $31^{\circ} \mathrm{C}$. La TCV y TCGA a las $24 \mathrm{HDE}$ fue significativamente mayor $(P<0,05)$ en las larvas a $31^{\circ} \mathrm{C}$. Sin embargo, a las $48 \mathrm{HDE}$ no se presentaron diferencias significativas $(P>$ $0,05)$ en la tasa de consumo de las reservas endógenas entre las larvas a 31 y $28^{\circ} \mathrm{C}$. A las $72 \mathrm{HDE}$ las larvas cultivadas a $28^{\circ} \mathrm{C}$ consumieron totalmente su vitelo y gota de aceite. Dado que las larvas a 25 y $31{ }^{\circ} \mathrm{C}$ no sobrevivieron a las 72 $\mathrm{HDE}$, solo se muestra la relación empírica entre el volumen del vitelo y de la gota de aceite de las larvas a $28^{\circ} \mathrm{C}$ durante

Tabla 1. Porcentaje y tiempo de eclosión (horas después de la fertilización, HDF) y de la longitud total de las larvas de $L$. guttatus en las diferentes temperaturas evaluadas y horas después de la eclosión (HDE). Valores del promedio \pm desviación estándar/ Percentage and hatching time (hours after fertilization, HDF) and total length of $L$. guttatus larvae at different evaluated temperatures and hours after hatch (HDE). Mean \pm standard deviation values

\begin{tabular}{lcccc}
\hline & \multicolumn{4}{c}{ Temperaturas $\left({ }^{\circ} \mathrm{C}\right)$} \\
\cline { 2 - 5 } & 21 & 25 & 28 & \multicolumn{1}{c}{31} \\
\hline Porcentaje de eclosión & ND & $83,1 \pm 16,0$ & $84,3 \pm 10,5$ & $96,1 \pm 1,3$ \\
Tiempo de eclosión (HDF)* & ND & $19,6 \pm 0,57^{\mathrm{a}}$ & $15,6 \pm 0,57^{\mathrm{b}}$ & $13,3 \pm 0,5^{\mathrm{c}}$ \\
LT al eclosionar (mm)* & & $1,67 \pm 0,13^{\mathrm{a}}$ & $1,77 \pm 0,16^{\mathrm{ab}}$ & $1,84 \pm 0,27^{\mathrm{b}}$ \\
LT a 24 HDE* & & $2,38 \pm 0,13^{\mathrm{a}}$ & $2,44 \pm 0,23^{\mathrm{ab}}$ & $2,48 \pm 0,09^{\mathrm{b}}$ \\
LT a 48 HDE* & & $\mathrm{ND}$ & $2,44 \pm 0,09^{\mathrm{a}}$ & $2,54 \pm 0,09^{\mathrm{b}}$ \\
LT a 72 HDE & & & $2,6 \pm 0,1$ & ND \\
\hline
\end{tabular}

ND: no determinado; *diferentes superíndices indican diferencias significativas entre tratamientos $(P<0,05)$ 
Tabla 2. Promedios \pm desviación estándar de volumen y consumo del saco vitelino (VSV, TCV) y de la gota de aceite (VGA, TCGA), en las larvas de $L$. guttatus obtenidas en las diferentes temperaturas evaluadas / Means \pm standard deviation of volume and yolk sac consumption (VSV, TCV) and oil droplet (VGA, TCGA) of L. guttatus larvae obtained in the different evaluated temperatures

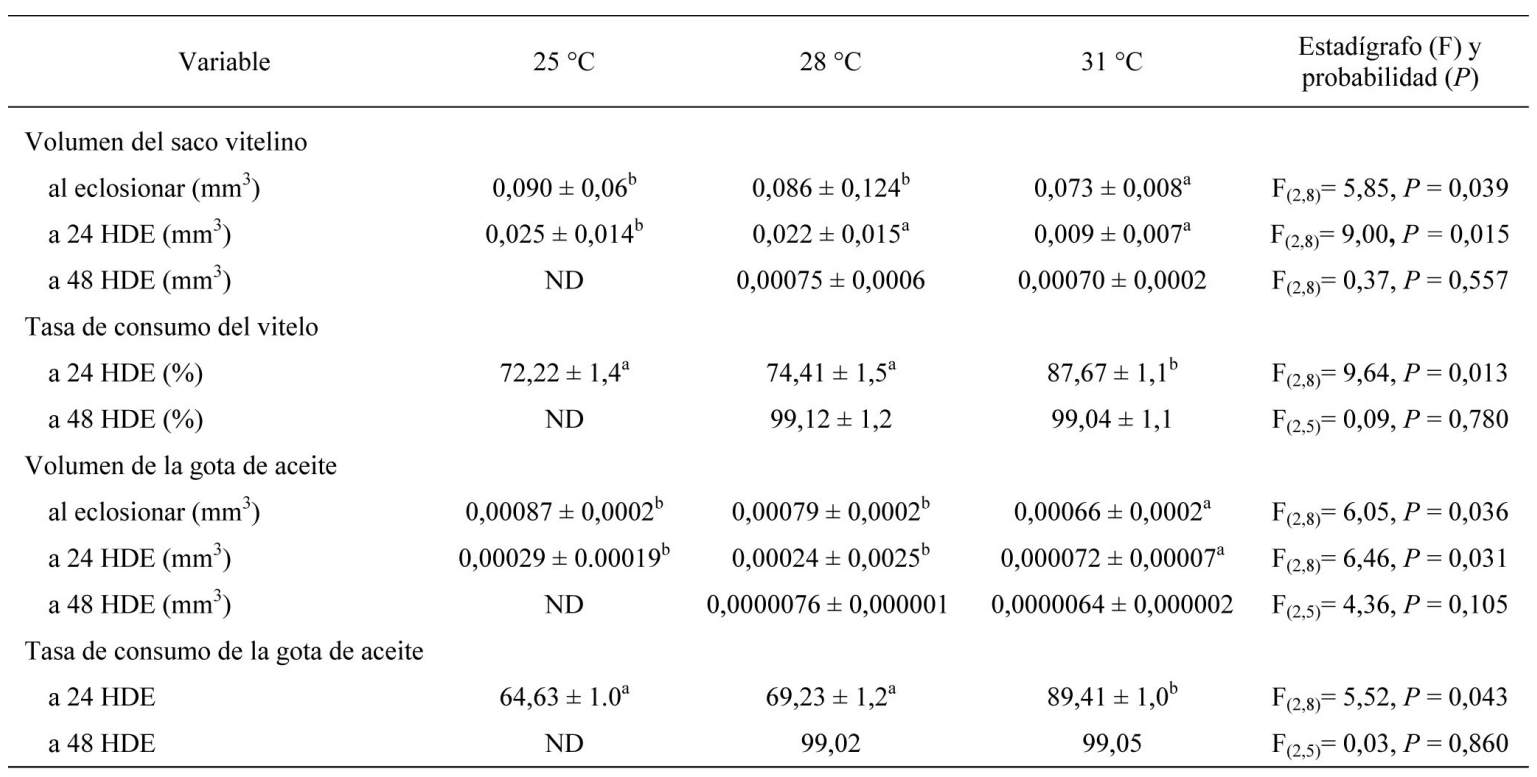

ND: no determinado; Los superíndices diferentes de una variable entre tratamientos indican diferencias significativas $(P<0,05)$

el tiempo experimental. El modelo que mejor se ajustó a los resultados obtenidos en relación al consumo de vitelo fue el de raíz cuadrada-X (Fig. 1A) con una $\mathrm{r}^{2}=0,939$; para el consumo de la gota de aceite el modelo mejor ajustado fue el de Y-inversa $\mathrm{X}^{2}$ (Fig. 1B) con una $\mathrm{r}^{2}=0,907$.

\section{DisCUSIÓN}

Los resultados del presente estudio demostraron que la temperatura de incubación tiene un efecto sobre el desarrollo y eclosión de los embriones de L. guttatus; a alta temperatura $\left(31^{\circ} \mathrm{C}\right)$ se incrementó el desarrollo del embrión y se aceleró el proceso de eclosión, mientras que a baja temperatura $\left(22^{\circ} \mathrm{C}\right)$, el embrión empezó a desarrollarse en las primeras horas después de la fertilización; pero antes de la eclosión cesó su desarrollo y hubo 100\% de mortalidad. La literatura señala que los estadios tempranos de los huevos de peces marinos antes de que el embrión esté completamente formado, son sensibles a los cambios del medio ambiente como temperatura, salinidad y oxígeno disuelto en el agua de cultivo; dentro de estos factores abióticos, la temperatura es determinante en el desarrollo de los embriones y larvas de peces, afectando tanto la eclosión como la supervivencia (Stickney 2000, Jaroszewska \& Dabrowski 2011). A bajas temperaturas $\left(17-22^{\circ} \mathrm{C}\right)$, los embriones de las especies subtropicales o tropicales no completan su desarrollo y no eclosionan, o eclosionan con muy baja supervivencia y presentan malformaciones, y a temperaturas altas (30-32 ${ }^{\circ} \mathrm{C}$ ) se acelera la eclosión (Kamler 1992, Gracia-López et al.
2004, Shi et al. 2010, Brown et al. 2011, Abdo-de la Parra et al. 2012, Ahn et al. 2012, Peña et al. 2014). En trabajos previos con L. guttatus se ha reportado que dependiendo de la temperatura, la eclosión se inicia entre las 15 y 17 HDF, concluyendo 20 y 21 HDF (Boza-Abarca et al. 2008, Abdo-de la Parra et al. 2010, Ibarra-Castro et al. 2012). En este trabajo, el porcentaje de eclosión fue similar a 25, 28 y $31^{\circ} \mathrm{C}$; lo cual coincide con lo reportado para L. peru (Peña et al. 2014, Estrada-Godínez et al. 2015) y Mycteroperca rocacea (Gracia-López et al. 2004) los cuales mostraron altos porcentajes de eclosión entre 24 y $30{ }^{\circ} \mathrm{C}$; de lo que se concluyó que la temperatura en que son mantenidos los reproductores, antes y durante el desove, puede determinar la tolerancia a la temperatura para los huevos desovados.

El tamaño de las larvas de peces al momento de la absorción total del saco vitelino, cuando se cambia de alimentación endógena a exógena, es muy importante para su supervivencia, ya que las larvas de mayor tamaño durante este periodo de transición pueden aumentar su capacidad para competir por el alimento y escapar de depredadores (Blaxter \& Hempel 1966, Heming \& Buddington 1988). Se ha demostrado que la tasa de crecimiento en las larvas de peces se acelera al aumentar la temperatura (Kaminski et al. 2006, Yoseda et al. 2006). En el presente estudio las larvas de pargo flamenco presentaron mayor longitud corporal (LT) a mayor temperatura; sin embargo la supervivencia también fue afectada por este parámetro; en las temperaturas extremas evaluadas $\left(25\right.$ y $\left.31^{\circ} \mathrm{C}\right)$ las larvas no sobrevivieron 
A

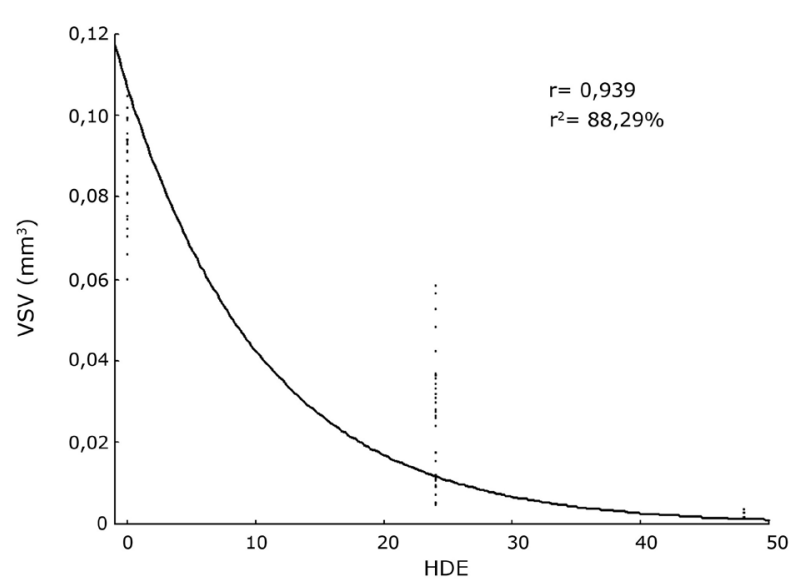

B

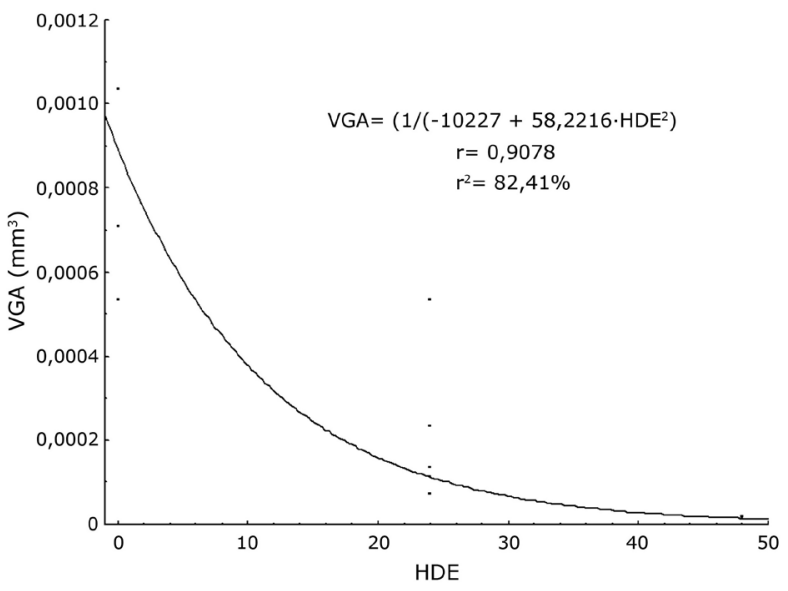

Figura 1. A) Volumen del saco vitelino (VSV) de las larvas de $L$. guttatus a $28^{\circ} \mathrm{C}$ a diferentes horas después de la eclosión (HDE); B) Volumen de la gota de aceite (VGA) de las larvas de $L$. guttatus a $28^{\circ} \mathrm{C}$ a diferentes horas después de la eclosión (HDE) / A) Yolk sac volume (VSV) of L. guttatus larvae at $28^{\circ} \mathrm{C}$ and different hours post hatching (HDE); B) Oil drop volume (VGA) of L. guttatus larvae under $28^{\circ} \mathrm{C}$ at different hours post hatching (HDE)

después de las 48 y $72 \mathrm{HDE}$, respectivamente; lo cual también ha sido observado en otras especies de peces dado que están fuera del rango de tolerancia de los organismos (Shi et al. 2010, Prokešová et al. 2015).

Tanto la tasa de absorción como la eficiencia de utilización del vitelo son determinantes en el desarrollo inicial, crecimiento y supervivencia de las larvas de peces. Estos procesos metabólicos son afectados por un número de factores tales como la temperatura, la luz, la concentración de oxígeno y la salinidad (Heming \& Buddington 1988). La absorción del vitelo en peces se da mediante una estructura especializada llamada sincitio, que desaparece una vez que el vitelo haya sido agotado (Zavala 2011). De manera general, el consumo de vitelo se ha caracterizado en muchos teleósteos por presentar 3 fases de absorción. La primera fase, llamada fase de pre-eclosión, es caracterizada por un suave pero constante incremento en la tasa de absorción de vitelo, donde el saco de vitelo y el glóbulo de aceite son consumidos en aproximadamente la misma proporción (Kamler 2008). Un poco antes del momento de la eclosión, la tasa de absorción de vitelo se incrementa rápidamente, probablemente en respuesta al aumento de la superficie de absorción debido a los cambios en la forma del saco vitelino o bien al aumento en la actividad metabólica del sincitio (Kamler 1992). Esto marca el inicio de la segunda fase o fase posteclosión, y es caracterizada por una relativamente alta y constante tasa de absorción. Dado que se aproxima el agotamiento del saco de vitelo, su tasa de absorción disminuye, probablemente en respuesta a la disminución de la superficie del área de absorción por el encogimiento del saco de vitelo o al cambio en la composición del vitelo (Kamler 2008). De esta forma da inicio de la tercera fase o fase terminal de absorción, en la cual, el glóbulo de aceite es preferentemente consumido (Heming \& Buddington 1988). Este patrón de consumo de reservas vitelinas también fue observado en el presente trabajo; en las primeras $24 \mathrm{HDE}$, la tasa de consumo del vitelo fue superior al $70 \%$ en las larvas de las 3 temperaturas evaluadas; posteriormente la tasa de absorción fue más lenta. En cambio, la tasa de consumo de la gota de aceite fue menor las primeras $24 \mathrm{HDE}$ a 25 y 28 ${ }^{\circ} \mathrm{C}$ (64 y 69\%, respectivamente) comparada con la tasa de consumo de vitelo. Este mismo patrón se ha reportado para diferentes especies como Chanos chanos, Lates calcarifer, Siganus guttatus (Bagarinao, 1986); Lutjanus campechanus (Williams et al. 2004), Pagrus pagrus (Aristizabal 2006), L. peru (Peña et al. 2014, Estrada-Godínez et. al. 2015), C. undecimalis (Barón-Aguilar et al. 2015), M. merluccius (Guevara-Fletcher et al. 2017), entre otras. Las proteínas del vitelo se consumen más rápido que otros nutrientes para desarrollar funciones específicas como formación de tejido y los lípidos se preservan como reserva de energía y reguladores hormonales (Kamler 2008). Los factores que alteren la actividad metabólica del sincitio también afectan, de manera directa, la tasa de absorción del vitelo, la cual, se incrementa como consecuencia de temperaturas fuera del rango óptimo de la especie (Heming \& Buddington 1988, Kamler 2002, Pittman et al. 2013). Además, el efecto de la temperatura sobre la eficiencia en la utilización del vitelo no presenta un patrón definido ya que la temperatura es especie-específico. Por ejemplo, las larvas de la cabrilla sardinera (Gracia-López et al. 2004), robalo (Barón-Aguilar et al. 2015) y huachinango (Peña et al. 2014, EstradaGodínez et. al. 2015), presentaron una mayor longitud a baja temperatura, mientras que, el consumo de vitelo y gota 
de aceite fue mayor a temperaturas altas. En otras especies como la merluza (Guevara-Fletcher et al. 2017) la mayor longitud se presentó en la temperatura más alta así como el consumo de vitelo y gota de aceite, similar a los casos del presente estudio; sin embargo, existen otros factores que podrían afectar los parámetros evaluados en este estudio, como por ejemplo, el choque térmico que pudieron recibir los huevos al momento de la transferencia del tanque de colecta al sistema de experimentación (Prokešová et al. 2015).

Este trabajo, demostró el efecto de la temperatura sobre el tiempo de eclosión, talla, supervivencia, consumo del vitelo y gota de aceite de las larvas de Lutjanus guttatus. Los resultados obtenidos sugieren que la temperatura para la incubación y cultivo de larvas vitelinas del pargo flamenco se debe llevar a cabo a los $28^{\circ} \mathrm{C}$ para lograr la sobrevivencia del embrión, eclosión, crecimiento y consumo adecuado de sus reservas energéticas y puedan lograr una exitosa transición a la alimentación exógena.

\section{Agradecimientos}

A V. Williams por su apoyo en la redacción del resumen en inglés y corrección del manuscrito. A J. Huerta por su asistencia técnica. Al personal de la planta piloto para la producción de peces marinos del CIAD, Mazatlán por proporcionar los huevos de pargo flamenco.

\section{LITERATURA CITADA}

Abdo-de la Parra MI, LE Rodríguez-Ibarra, F CampilloMartínez, G Velasco-Blanco, N García-Aguilar, L Álvarez-Lajonchère \& D Voltolina. 2010. Efecto de la densidad de siembra sobre el crecimiento y supervivencia larval del pargo lunarejo Lutjanus guttatus (Steindachner, 1869). Revista de Biología Marina y Oceanografía 45(1): 141-146.

Abdo-de la Parra MI, I Martínez-Rodríguez, B GonzálezRodríguez, E Rodríguez-Ibarra, N Duncan \& C Hernández. 2012. Efecto de la temperatura y salinidad del agua en la incubación de huevos de botete diana Sphoeroides annulatus. Revista de Biología Marina y Oceanografía 47(1): 147-153.

Ahn H, Y Yamada, A Okamura, N Hori, N Mikawa, S Tanaka \& K Tsukamoto. 2012. Effect of water temperature on embryonic development and hatching time of the Japanese eel Anguilla japonica. Aquaculture 330/333: 100-105.

Álvarez-Lajonchère, LS, MI Abdo-de la Parra, LE Rodríguez-Ibarra, G Velasco-Blanco, A Puello-Cruz, B González-Rodríguez, A Ibarra-Soto \& L Ibarra-Castro. 2012. The scale-up of spotted rose snapper, Lutjanus guttatus, larval rearing at Mazatlan, Mexico. Journal of World Aaquaculture Society 43(3): 411-421.

Aristizabal OE. 2006. Consumo de oxígeno, crecimiento y utilización del alimento durante el desarrollo larval del besugo Pagrus pagrus (Linné, 1758) (Pisces: Sparidae). Revista de Biología Marina y Oceanografía 41(2): 209-222.
Azocar C, G Claramunt, F Yañez \& M Futagawa. 2014. Efecto de la temperatura sobre el desarrollo embrionario y larval de Graus nigra (Kyphosidae) del norte de Chile. Revista de Biología Marina y Oceanografía 49(1): 111-122.

Bagarinao T. 1986. Yolk resorption, onset of feeding and survival potential of larvae of three tropical marine fish species reared in hatchery. Marine Biology 91: 449-459

Barón-Aguilar C, C Rhody, NP Bremman, KL Main, EB Peebles \& FE Muller-Karger. 2015. Influence of temperature on yolk resorption in common snook Centropomus undecimalis (Bloch, 1792) larvae. Aquaculture Research 46: 1679-1687.

Blaxter JHS. 1992. The effect of temperature on larval fishes. Netherlands Journal of Zoology 42: 336-357.

Blaxter JHS \& G Hempel. 1966. Utilization of yolk by herring larvae. Journal of Marine Biology 46: 219-234.

Boza-Abarca J, E Calvo-Vargas, N Solís-Ortiz \& J Komen. 2008. Induced spawning and larval rearing of spotted rose snapper, Lutjanus guttatus, at the Marine Biology Station, Puntarenas, Costa Rica. Ciencias Marinas 34: 239-252.

Brown CA, CT Gothreaux \& CC Green. 2011. Effects of temperature and salinity during incubation on hatching and yolk utilization of Gulf killifish Fundulus grandis embryos. Aquaculture 315: 335-339.

Das TS, AK Pal, SK Chakraborty, M Manush, RS Dalvi, K Sarma \& SC Mukherjee. 2006. Thermal dependence of embryonic development and hatching rate in Labeo rohita (Hamilton 1822). Aquaculture 255: 536-541.

Estrada-Godínez JA, LD Moreno-Figueroa, M MaldonadoGarcía, JC Pérez-Urbiola, J Romero-Rodríguez \& A Audelo-Naranjo. 2015. Influence of the temperature on the early larval development of the Pacific red snapper, Lutjanus peru (Nichols \& Murphy, 1922). Latin American Journal of Aquatic Research 43(1): 137-145.

Gracia-López V, M Kiewek-Martínez \& M MaldonadoGarcía. 2004. Effects of temperature and salinity on artificially reproduced eggs and larvae of the leopard grouper Mycteroperca rosacea. Aquaculture 237: 485-498.

Guevara-Fletcher C, O Álvarez, J Sánchez \& J Iglesias. 2017. The effect of temperature on the development of yolk-sac larvae of European hake (Merluccius merluccius L.) under laboratory conditions. Aquaculture Research 48(4): 1392-1405.

Hardy RS \& M Livak. 2004. Effects of temperature on the early development, growth and survival of shortnose sturgeon, Acipenser brevirostrum, and Atlantic sturgeon, Acipenser oxyrhynchus, yolk sac larvae. Environmental Biology of Fishes 70: 145-154.

Heming TA \& RK Buddington. 1988. Yolk absorption in embryonic and larval fishes. In: Hoar WS \& DJ Randall (eds). Fish physiology. Vol. XI. The physiology of developing fish. Part A. Eggs and larvae, pp. 408-447. Academic Press, San Diego.

Hui W, L Guodong, L Jiahui, Y Hongshuai, Q Jun \& X Pao. 2015. Combined effects of temperature and salinity on yolk utilization in Nile tilapia (Oreachromis niloticus). Aquaculture Research 46: 2418-2425. 
Ibarra-Castro L \& L Álvarez-Lajonchère. 2011. GnRhainduced multiple spawns and volition spawning of captive Spotted Rose Snapper, Lutjanus guttatus, at Mazatlan, Mexico. Journal of the World Aquaculture Society 42(4): 564-574.

Ibarra-Castro L, L Muñoz-Meza \& L Álvarez-Lajonchère. 2012. Estudios sobre el manejo e incubación de huevos del pargo flamenco Lutjanus guttatus (Pisces, Lutjanidae). Hidrobiológica 22(1): 49-57.

Jaroszewska M \& K Dabrowski. 2011. Utilization of yolk: transition from endogenous to exogenous nutrition in fish. In: Holt GJ (ed). Larval fish nutrition, pp. 183-218. John Wiley \& Sons, Chichester.

Kaminski R, E Kamler E, M Korwin-Kossakowsk, L Myszkowski \& J Wolnicki. 2006. Effects of different incubation temperatures on the yolk-feeding stage of Eupallasella percnurus (Pallas). Journal of Fish Biology 68: 1077-1090.

Kamler E. 1992. Early life history of fish. An energetics approach, 267 pp. Chapman and Hall, London.

Kamler E. 2002. Ontogeny of yolk-feeding fish: an ecological perspective. Reviews in Fish Biology and Fisheries 12: 79-103.

Kamler E. 2008. Resource allocation in yolk-feeding fish. Reviews in Fish Biology and Fisheries 18: 143-200.

Peña R, S Dumas, I Zavala-Leal \& M Contreras-Olguín. 2014. Effect of incubation temperature on the embryonic development and yolk-sac larvae of the Pacific red snapper Lutjanus peru (Nichols \& Murphy, 1922). Aquaculture Research 45: 519-527.
Pittman K, M Yúfera, M Pavlidis, AJ Geffen, W Koven, L Ribeiro, JL Zambonino-Infante \& A Tandler. 2013. Fantastically plastic: fish larvae equipped for a new world. Reviews in Aquaculture 5(1): 224-267.

Prokešová B, B Drozd, J Kouril, V Stejskal \& J Matousek. 2015. Effect of water temperature on early life history of African sharp-tooth catfish, Clarias gariepinus (Burchell, 1822). Journal of Applied Ichthyology 31(2): 18-29.

Shi Y-H, G-Y Zhang, Y-Z Zhu, J-Z Liu \& W-L Zang. 2010. Effects of temperature on fertilized eggs and larvae of tawny puffer Takifugu flavidus. Aquaculture Research 41(12): 1741-1747.

Stickney RR. 2000. Temperature. In: Stickney RR (ed). Encyclopedia of aquaculture, pp. 929-934. John Willey \& Sons, New York.

Williams K, N Papanikos, RP Phelps \& JD Shardo. 2004. Development, growth, and yolk utilization of hatcheryreared red snapper Lutjanus campechanus larvae. Marine Ecology Progress Series 275: 231-239.

Yoseda K, S Dan, T Sugaya, K Yokogi, M Tanaka \& S Tawada. 2006. Effects of temperature and delayed initial feeding on the growth of Malabar grouper (Epinephelus malabaricus) larvae. Aquaculture 256: 192-200.

Zavala I. 2011. Caracterización bioquímica del consumo de reservas vitelinas en peces teleósteos de ontogenia indirecta. Revista Electrónica de Veterinaria 12(3): 1-32. 